\title{
Mucolipin 2 is differentially expressed in lymph node metastasis in human breast cancer.
}

Shahan Mamoor, MS ${ }^{1}$

${ }^{1}$ shahanmamoor@gmail.com

East Islip, NY USA

Metastasis to the brain is a clinical problem in patients with breast cancer ${ }^{1-3}$. Between the breast and the brain reside the secondary lymphoid organ, the lymph nodes. We mined published microarray data ${ }^{4,5}$ to compare primary and metastatic tumor transcriptomes for the discovery of genes associated with metastasis to the lymph nodes in humans with metastatic breast cancer. We found that mucolipin 2, encoded by MCOLN2, was among the genes whose expression was most different in the lymph node metastases of patients with metastatic breast cancer as compared to primary tumors of the breast. MCOLN2 mRNA was present at increased quantities in lymph node metastases as compared to primary tumors of the breast. Importantly, expression of MCOLN2 in primary tumors of the breast was correlated with patient distant metastasis-free survival, in lymph node positive patients but not in lymph node negative patients. Modulation of MCOLN2 expression may be relevant to the biology by which tumor cells metastasize from the breast to the lymph nodes and the brain in humans with metastatic breast cancer.

Keywords: breast cancer, metastasis, brain metastasis, central nervous system metastasis, lymph node metastasis, mucolipin 2 , MCOLN2, systems biology of breast cancer, targeted therapeutics in breast cancer. 
One report described a 34\% incidence of central nervous system metastases in patients treated with trastuzumab for breast cancer ${ }^{2}$. More recently, the NEfERT-T clinical trial ${ }^{6}$ which compared administration of either neratinib or trastuzumab in conjunction with paclitaxel demonstrated that in a randomized, controlled setting, in breast cancer patients treated with neratinib, not only was the incidence of central nervous system recurrence significantly lower, the time to central nervous system metastasis was significantly delayed as compared to patients administered trastuzumab ${ }^{6}$. The alarmingly high rate of central nervous system metastasis described, as well as data, both anecdotal ${ }^{2}$ and from a randomized, controlled setting ${ }^{6}$ illustrating that treatment with trastuzumab may be associated with these events demands an enhanced understanding of the transcriptional makeup of brain and lymph node metastatic tissues to support identification of therapeutic targets, whether they are treatment related or not. The lymph nodes reside between the breast and the brain. We performed a global comparative analysis of lymph node metastatic tumor tissues in patients with metastatic breast cancer, compared to primary tumors and normal breast tissues ${ }^{4,5}$. We discovered transcriptome-wide differential expression of the gene encoding mucolipin 2, MCOLN2, in lymph node metastatic tissues of patients with metastatic breast cancer.

\section{Methods}

We used datasets GSE10893 ${ }^{4}$ and GSE42568 ${ }^{5}$ for this global differential gene expression analysis of brain metastatic breast cancer in conjunction with GEO2R. GSE10893 was generated using Agilent-011521 Human 1A Microarray G4110A technology with $n=71$ primary breast tumors and $n=7$ lymph node metastases from patients with breast cancer; analysis was performed using platform GPL887. GSE42568 was generated using Affymetrix Human Genome U133 Plus 2.0 array technology with $n=17$ normal breast tissue biopsies and $n=104$ primary breast tumor biopsies from patients with breast cancer; analysis was performed using platform GPL570. The Benjamini and Hochberg method of $p$-value adjustment was used for ranking of differential expression but raw $p$-values were used to assess statistical significance of global differential expression. Log-transformation of data was auto-detected, and the NCBI generated category of platform annotation was used. A statistical test was performed to evaluate whether MCOLN2 gene expression was significantly different between primary tumors of the breast and lymph node metastases in humans with breast cancer using a two-tailed t-test. For Kaplan-Meier survival analysis, we used the Kaplan-Meier plotter online tool ${ }^{7}$ for correlation of MCOLN2 mRNA expression levels with distant metastasis-free survival in $n=2032$ breast cancer patients, in $n=261$ lymph node positive breast cancer patients and in $n=240$ lymph node negative breast cancer patients.

\section{$\underline{\text { Results }}$}

We performed global comparative transcriptome analysis of metastatic tumor tissues of patients with metastatic breast cancer using published microarray data ${ }^{4,5}$ to describe the transcriptional landscape of metastasis in human breast cancer in an unbiased fashion and for the discovery of novel therapeutic targets.

\section{MCOLN2 is differentially expressed in the lymph node metastases of patients with metastatic breast cancer.}

Through blind, systems-level analysis of published microarray data ${ }^{4}$, we identified mucolipin 2, encoded by MCOLN2, as a differentially expressed gene in the lymph node metastatic tissues of humans with breast cancer (Table 1). When sorting each of the genes expressed in lymph node metastases based on significance of difference as compared to primary tumors of the breast in patients with breast cancer, MCOLN2 ranked 202 out of 18034 total transcripts (Chart 1), equating to 98.9\% differential expression. Differential expression of MCOLN2 in the lymph node metastases of patients with metastatic breast cancer was statistically significant (Chart $1 ; p=2.44 \mathrm{e}-03$ ). 
To validate transcriptome-wide differential expression of MCOLN2 in human breast cancer, we queried a second microarray dataset ${ }^{5}$, here comparing normal breast tissues and primary tumors of the breast. Here, we again identified MCOLN2 as a differentially expressed in the brain metastatic tissues of patients with breast cancer (Chart 2). When sorting each of the genes expressed in brain metastases based on significance of difference as compared to normal breast tissues, MCOLN2 ranked 14568 out of 54675 total transcripts (Chart 2), equating to $73.4 \%$ differential expression. Differential expression of MCOLN2 in the primary tumors of patients with breast cancer was not statistically significant (Chart $2 ; p=6.59 \mathrm{e}-03$ ). Thus, transcriptome-wide differential expression of MCOLN2 in the tumor tissues of women with breast cancer was conserved across two independent microarray datasets, both in primary and metastatic tumor tissues.

\section{MCOLN2 is expressed at higher levels in the lymph node metastases of patients with metastatic breast cancer.}

We obtained exact mRNA expression levels for MCOLN2, in primary tumors of the breast and in lymph node metastases of patients with metastatic breast cancer to determine direction and statistical significance of change in MCOLN2 expression in lymph node metastatic tissues. We observed significantly increased expression of MCOLN2 in the lymph node metastases of patients with breast cancer as compared to primary tumors of the breast: MCOLN2 was expressed at $0.32 \pm 0.49$ arbitrary units (AU) in primary tumors of the breast, while it was expressed at $0.93 \pm 0.46 \mathrm{AU}$ in lymph node metastatic tissues (Figure 1). The difference in MCOLN2 mRNA levels between primary tumors of the breast and lymph node metastases was statistically significant (Figure $1 ; p=0.002583$ ).

\section{MCOLN2 expression is significantly correlated with survival outcomes in human breast cancer.}

We performed Kaplan-Meier survival analysis ${ }^{7}$ in $n=261$ lymph node positive breast cancer patients and $n=240$ lymph node negative breast cancer patients, to evaluate whether MCOLN2 tumor expression was correlated with survival outcomes in breast cancer and to evaluate whether lymph node status influenced correlation of MCOLN2 tumor expression with survival outcomes in human breast cancer. We observed a correlation between primary tumor expression of MCOLN2 and distant metastasis-free survival (DMFS) in lymph node positive breast cancer (Figure 2). Lymph node positive patients whose primary tumors expressed low levels of MCOLN2 possessed median DMFS of 79.46 months as compared to a median DMFS of 30.98 months in lymph node positive patients whose tumors expressed high levels of MCOLN2. This difference in DMFS based on MCOLN2 tumor expression in lymph node positive patients with breast cancer trended towards statistical significance (Figure 2, Chart 3; logrank $p$-value: 0.08 (hazard ratio: $1.53(0.95-2.47)$ )). MCOLN2 primary tumor expression was not (Figure 2, Chart 3; logrank $p$-value: 0.53 ; hazard ratio: $1.25(0.62-2.5)$ ).

Thus, by mining published microarray data ${ }^{4,5}$ in an unbiased and systematic fashion, we identified MCOLN2 as among the genes whose expression was most different, transcriptome-wide, in the lymph node metastases of patients with breast cancer, when compared to primary tumors of the breast, we observed significantly increased expression of MCOLN2 in lymph node metastases as compared to primary tumors of the breast. Further, we found a significant correlation between MCOLN2 expression and patient survival outcomes, as primary tumor expression of MCOLN2 was correlated with distant metastasis-free survival in lymph node positive patients but not in lymph node negative patients. 


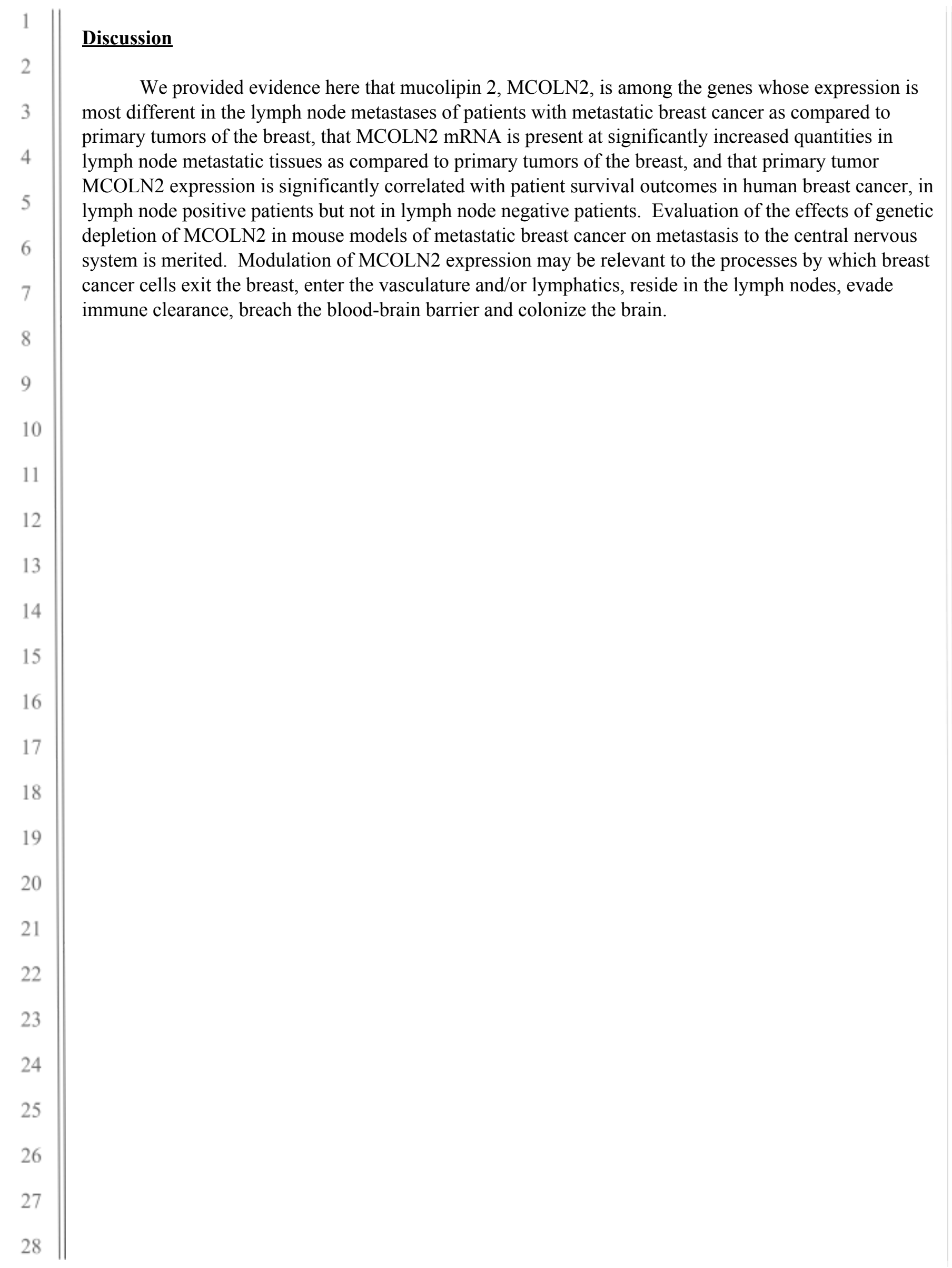




\section{References}

1. Lin, N.U., Amiri-Kordestani, L., Palmieri, D., Liewehr, D.J. and Steeg, P.S., 2013. CNS metastases in breast cancer: old challenge, new frontiers.

2. Bendell, J.C., Domchek, S.M., Burstein, H.J., Harris, L., Younger, J., Kuter, I., Bunnell, C., Rue, M., Gelman, R. and Winer, E., 2003. Central nervous system metastases in women who receive trastuzumab-based therapy for metastatic breast carcinoma. Cancer, 97(12), pp.2972-2977.

3. Tsukada, Y., Fouad, A., Pickren, J.W. and Lane, W.W., 1983. Central nervous system metastasis from breast carcinoma autopsy study. Cancer, 52(12), pp.2349-2354.

4. Weigman, V.J., Chao, H.H., Shabalin, A.A., He, X., Parker, J.S., Nordgard, S.H., Grushko, T., Huo, D., Nwachukwu, C., Nobel, A. and Kristensen, V.N., 2012. Basal-like Breast cancer DNA copy number cancer research and treatment, 133(3), pp.865-880.

5. Clarke, C., Madden, S.F., Doolan, P., Aherne, S.T., Joyce, H., O'driscoll, L., Gallagher, W.M., Hennessy, B.T., Moriarty, M., Crown, J. and Kennedy, S., 2013. Correlating transcriptional networks to breast cancer survival: a large-scale coexpression analysis. Carcinogenesis, 34(10), pp.2610-2618.

6. Awada, A., Colomer, R., Inoue, K., Bondarenko, I., Badwe, R.A., Demetriou, G., Lee, S.C., Mehta, A.O., Kim, S.B., Bachelot, T. and Goswami, C., 2016. Neratinib plus paclitaxel vs trastuzumab plus paclitaxel in previously untreated metastatic ERBB2-positive breast cancer: the NEfERT-T randomized clinical trial. JAMA oncology, 2(12), pp.1557-1564.

7. Györffy, B., Lanczky, A., Eklund, A.C., Denkert, C., Budczies, J., Li, Q. and Szallasi, Z., 2010. An online survival analysis tool to rapidly assess the effect of 22,277 genes on breast cancer prognosis using microarray data of 1,809 patients. Breast cancer research and treatment, 123(3), pp.725-731. 
Rank: 202

Probe ID: 3269

p-value: $2.44 \mathrm{e}-03$

t: -3.1324006

B: -1.562

Gene: MCOLN2

Gene name: mucolipin 2

Chart 1: MCOLN2 is differentially expressed in lymph node metastatic breast cancer when comparing lymph node metastases to primary tumors of the breast.

The rank of global differential expression, the probe/transcript ID, the $p$-value with respect to differential expression transcriptome-wide, $\mathrm{t}$, a moderated $\mathrm{t}$-statistic, $\mathrm{B}$, the log-odds of differential expression between the groups compared, the gene and gene name are listed in this chart.

Rank: 14568

Probe ID: 261110_at

p-value: $6.59 \mathrm{e}-03$

t: -2.77

B: -3.483251

Gene: MCOLN2

Gene name: mucolipin 2

Chart 2: MCOLN2 is differentially expressed in human breast cancer when comparing primary tumors of the breast to normal breast tissues.

The rank of global differential expression, the probe/transcript ID, the $p$-value with respect to differential expression transcriptome-wide, $\mathrm{t}$, a moderated t-statistic, $\mathrm{B}$, the log-odds of differential expression between the groups compared, the gene and gene name are listed in this chart. 


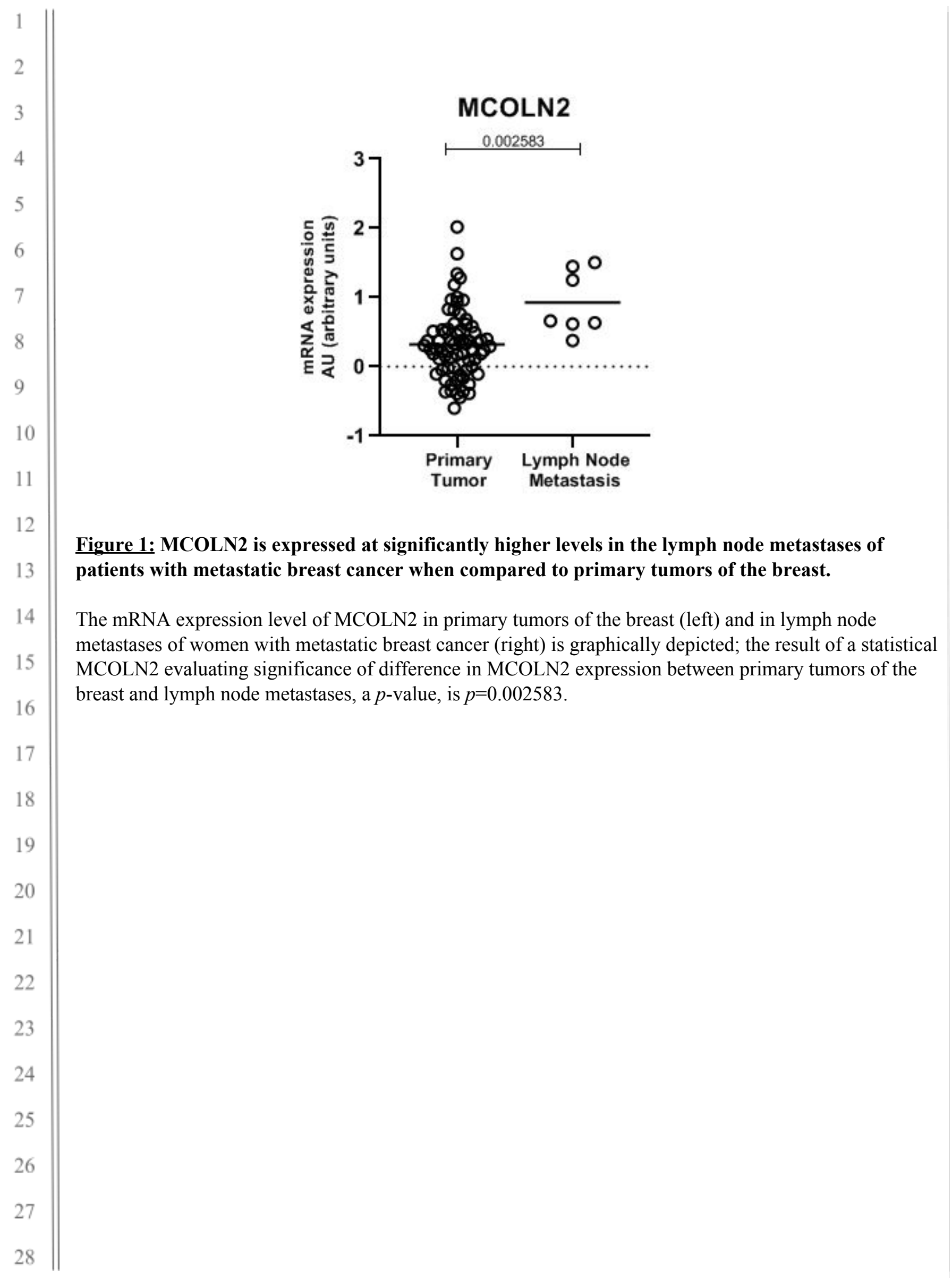



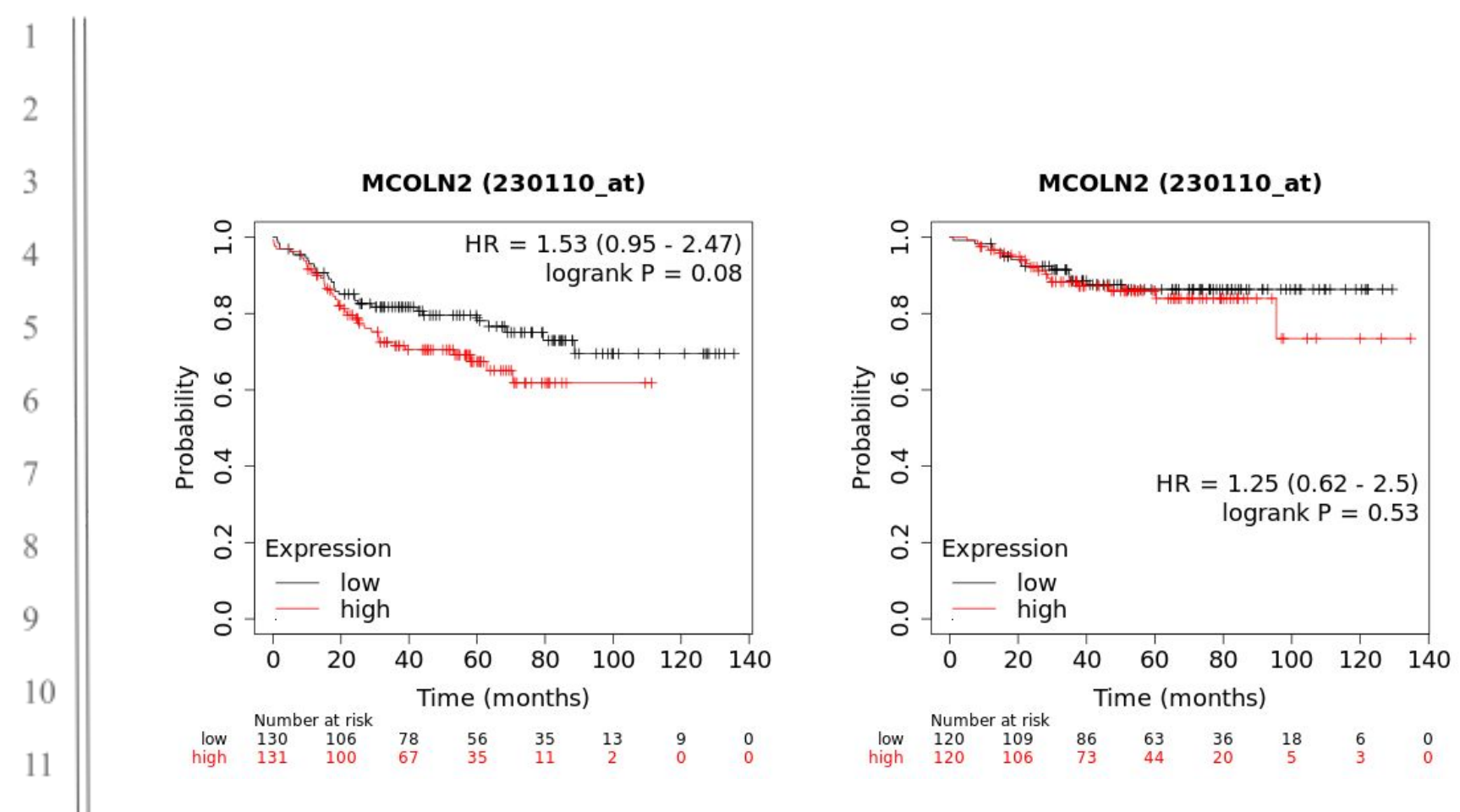

Figure 2: Significant correlation between MCOLN2 primary tumor expression and distant metastasis-free survival in lymph node positive patients but not in lymph node negative patients.

Depicted in these Kaplan-Meier plots is the probability of distant metastasis-free survival in $n=261$ lymph node positive patients (left) and $n=240$ lymph node negative patients (right), stratified into two groups, based on low or high expression of MCOLN2 in patient primary tumors. The log rank $p$-value denoting statistical significance of difference in distant metastasis-free survival when comparing the two groups, as well as hazard ratio for this comparison is listed above. Listed below is the number of patients at risk (number of patients alive) per interval, after stratification based on MCOLN2 expression; in the first interval, number at risk is number of patients alive; in each subsequent interval, number at risk is the number at risk less those who have expired or are censored. 


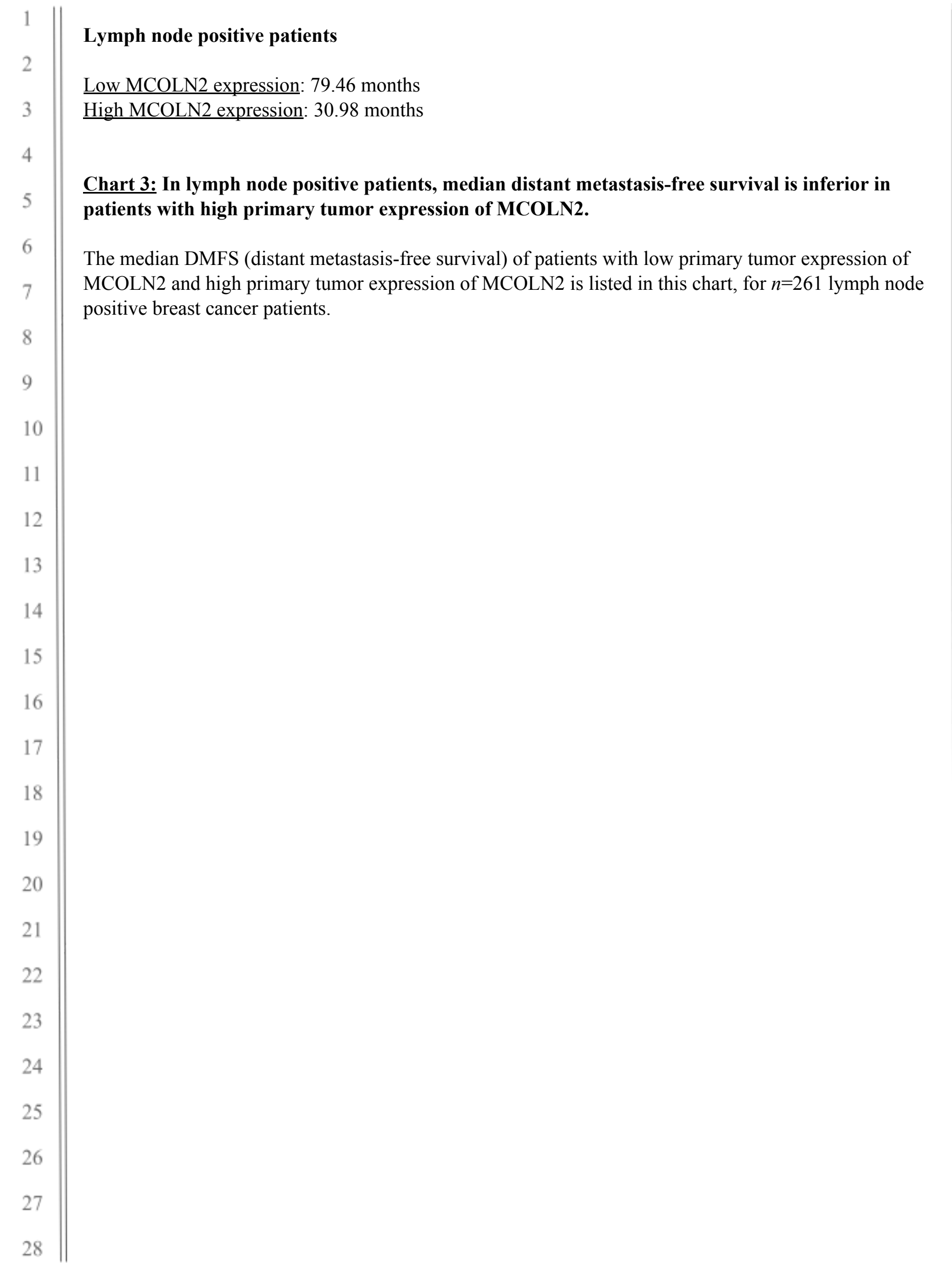

